Irish Math. Soc. Bulletin

Number 72, Winter 2013, 39-44

ISSN 0791-5578

\title{
WHY DO RESEARCH IN PURE MATHEMATICS?
}

\author{
STEPHEN M. BUCKLEY
}

ABstract. We discuss why people do research in pure mathematics, and its benefits to society.

The following short article is based on a webpage aimed at prospective postgraduate students created for the NUI Maynooth Mathematics and Statistics Department website. It is being presented here in a slightly adjusted form.

Many non-mathematicians have some sense of the importance of statistics and applied mathematics (the adjective "applied" being rather suggestive!), but they may view pure mathematics as something with little use. Consequently, we feel it is important to say a little about pure mathematics and the reasons for doing research in it. More precisely, we address the following basic questions:

- What is mathematics?

- Why do people do research in mathematics?

- What are the benefits to society of research in pure mathematics?

\section{WHAT IS MATHEMATICS?}

It is not possible to give an answer to this question that will satisfy all mathematicians, since so many fields in mathematics are so different from each other. Indeed, we are tempted to throw our hands up in the air and echo the words of Justice Potter Stewart by simply saying, "I know it when I see it." But if we wish to say something more informative than this, we could say that mathematics is the logical and abstract study of pattern.

\section{Why DO PEOPLE DO RESEARCH IN MATHEMATICS?}

One of the main reasons that mathematicians do research is because they appreciate the beauty of the particular types of abstract

Received on 28-12-2013. 
patterns involved in their own research, and enjoy discovering nonobvious aspects of these complex patterns.

Human beings are naturally quite good at recognizing all sorts of patterns, and through all our senses we seem to have an in-built fascination with pattern: we like to cover the inside of our houses with nicely patterned wallpaper or paintwork, and the outside with nicely patterned brickwork or other finishes, we like the complex patterns of music (whether that music is the latest popular music or something classical), and scientists in all fields get a thrill when a formerly unpredictable phenomenon is seen to be governed by some pattern. Evolutionary biologists would likely say that there are good evolutionary reasons for this: the weather, the seasons, habits of predators, and many other things exhibit patterns and, over the course of human evolution, it has been advantageous for us to be good at recognizing these patterns.

The fascination that mathematicians have for their research is thus arguably a mere extension of the fascination that all people have with pattern. When humans examine a complex pattern, whether by admiring the decorations of the Alhambra in Granada, or by listening to a fugue by Bach, they occasionally notice patterns that they had not noticed before, a phenomenon that they generally find delightful. This is precisely the reaction that a mathematician feels when he/she makes a breakthrough in understanding some complex abstract patterns.

Indeed, mathematics is analogous to an extra sense that many people lack. When our brains process, and make sense out of, the complicated array of words and symbols on a page of mathematics, we begin to understand the patterns and appreciate their beauty. That beauty has no more to do with the complicated sequence of symbols on the page than the beauty of the Alhambra has to do with the complex sequence of photons that hit our eyes when we look at the Alhambra's decorations. In both cases, the brain must do a substantial amount of processing to understand the complexity of the input data, and appreciate the patterns. Trying to explain the beauty of advanced mathematics to someone with little mathematical training is as difficult as trying to explain the beauty of the Alhambra to a blind person.

Although appreciation of the beauty of mathematics, and delight at discovering new patterns within complex abstract patterns is one 
of the primary motivations for all mathematicians, there are a variety of other reasons that people do research in mathematics. Indeed, some people may get as much enjoyment out of solving a crossword puzzle or getting a high score in a video game as a mathematician does in discovering something new in mathematics, but people typically do not make careers out of those other activities. The fact is that mathematics research is extremely useful!

\section{What ARE THE BENEFITS TO SOCIETY OF RESEARCH IN PURE MATHEMATICS?}

When we are teaching a child to count, we normally count a variety of different things with them: toys, Lego bricks, cartoon animals, and so on. The child eventually understands the abstract principle behind these concrete instances of number, and can then go on to count all the important things that need to be counted later in life. By understanding the abstract principles, they are well prepared not just for putting numbers to use in their everyday activities, but also for counting something new (such as scoops of baby formula when they themselves have a child). If a friend told us that they were asked:

Why are you teaching your child to count toys? Since they will likely be a bank teller like yourself, wouldn't it be better to teach them how to count money?

most of us would consider such a question ridiculous: we know at least subconsciously that number is an abstract concept, and that the counting of toys and cartoon animals is merely a means to the key goal of understanding number in the abstract. When we understand the abstract concept well, we can then put it to use in all sorts of areas.

Understanding a piece of abstract mathematical theory is like understanding numbers in the abstract. We usually gain the understanding by looking at special cases. And, although an abstract mathematical theory, just like the abstract concept of number, is divorced from the real world, it very often has the potential to be useful in a variety of different areas: its abstraction is a strength because it maximizes its potential usefulness. Or to quote Henri Poincaré [4, p.25], 
Mathematicians do not study objects, but the relations between objects; to them it is a matter of indifference if these objects are replaced by others, provided that the relations do not change. Matter does not engage their attention, they are interested by form alone.

One example of the power of abstraction is provided by Laplace's equation, one of the most studied and best understood (non-trivial) partial differential equations. A variety of phenomena in astronomy, electromagnetism, and fluid flow are governed by this equation, as is the steady state heat distribution in an object. By understanding the abstract mathematical equation, we simultaneously gain an understanding of all these phenomena.

CT scanners are one of the greatest advances in modern medical technology. These scanners form a three-dimensional image from a collection of two-dimensional images taken from different angles. The same principle is employed in reflection seismology to create three-dimensional images of the earth's subsurface, and in certain types of electron microscopy. In all cases, the underlying mathematics involves the inverse Radon transform, or related transforms. When Johann Radon and others investigated such transforms (from 1917 onwards), they were designed for applications within pure mathematics (specifically, harmonic analysis and related areas), but their importance outside mathematics was not recognised.

There are many other examples of mathematical research that for a long time seemed to have little relevance outside mathematics, but which eventually became matters of great importance there. The pursuit of a proof of Euclid's fifth postulate appeared increasingly quixotic as many people tried and failed over two millennia. When Farkas Bolyai, one of the mathematicians who tried and failed to resolve the issue, discovered that his son János had also begun to work on this problem, he wrote to János:

For God's sake, I beseech you, give it up. Fear it no less than sensual passions because it too may take all your time and deprive you of your health, peace of mind, and happiness in life.

But János persisted, and he and other 19th Century mathematicians made important breakthroughs that eventually led to modern manifold theory, which was central to Einstein's development of 
General Relativity. After Einstein's mathematician friend, Marcel Grossmann explained manifold theory to him, Einstein wrote ... in all my life I have not laboured nearly so hard, and I have become imbued with great respect for mathematics, the subtler part of which I had in my simplemindedness regarded as pure luxury until now.

Another example is provided by Number Theory, long considered the most inapplicable of all areas of mathematics. It was widely felt that the only reason to do research in Number Theory was to discover its beauty. This all changed with modern cryptography, which is an essential part of e-commerce and relies heavily on the properties of prime numbers and other aspects of Number Theory.

However the possible arrival of genuinely useful quantum computers in the not-too-distant future presents challenges for cryptography: due to the work of Shor [5], most popular public-key cryptographic methods would appear to be vulnerable to quantum computing algorithms. Post-quantum cryptography involves the development of other approaches that are not efficiently breakable using quantum computers. Such new methods depend on various parts of modern mathematics: for instance, ring theory is needed to develop lattice-based cryptographic schemes such as that proposed in [3].

For further related reading, we refer the reader to two wonderful philosophical articles about the usefulness of mathematics in the physical sciences and engineering. The first one, entitled The unreasonable effectiveness of mathematics in the natural sciences, was published by the physicist Eugene Wigner [6] in 1960. It argues that the way in which the mathematical structure of a physical theory often points the way to further advances in that theory and even to empirical predictions, is not a coincidence but must reflect some larger and deeper truth about both mathematics and Physics. A follow-up article [2] with a similar title, written by the Mathematician/Computer Scientist Richard Hamming in 1980, makes several attempts at answering Wigner's questions but, in the end, Hamming admits that all of his points combined are still insufficient to explain why mathematics is so useful and so often leads to further advances in other fields.

There are quite a few websites that describe a variety of examples of the applicability of mathematics; we recommend looking for instance at [7] and [8]. We also recommend the interesting account 
[1] by Kurt Bryan and Tanya Leise on the use of linear algebra in Google's PageRank algorithm.

We should add that the division between pure and applied mathematics is a rather false one. Any reasonable attempt to list the important areas of modern pure mathematics research would include many topics of great importance in applications. Conversely, an applied mathematician working, for instance, on efficient implementations of a numerical solution technique is often led naturally to study related abstract mathematical problems (for instance, the properties of certain special types of matrices).

Finally, outside of its direct applicability to the world around us, mathematical research helps us to improve and refresh the quality of what we teach, and certainly the world needs a large number of graduates with a wide variety of mathematical skills to fill the wide variety of positions that require some mathematics or the ability to analyse problems logically.

\section{REFERENCES}

[1] K. Bryan and T. Leise, The $\$ 25,000,000,000$ eigenvector: the linear algebra behind Google, SIAM Rev. 48 (2006), 569-581; available at http://www.rose-hulman.edu/ bryan/googleFinalVersionFixed.pdf (retrieved 2/12/2013).

[2] R.W. Hamming, The unreasonable effectiveness of mathematics, Amer. Math. Monthly 87 (1980), 81-90.

[3] T. Güneysu, V. Lyubashevsky, and T. Pöppelmann, Practical lattice-based cryptography: a signature scheme for embedded systems, Cryptographic Hardware and Embedded Systems - CHES 2012, 530-547, Lecture Notes in Computer Science 7428, Springer, Heidelberg, 2012.

[4] H. Poincaré, Science and hypothesis, Project Gutenberg EBook, 1911, available at ftp://sailor.gutenberg.lib.md.us/gutenberg/3/7/1/5/37157/ 37157-pdf.pdf

[5] P. Shor, Algorithms for quantum computation: discrete logarithms and factoring, 35th Annual Symposium on Foundations of Computer Science (Santa Fe, NM, 1994), 124-134, IEEE Comput. Soc. Press, Los Alamitos, CA, 1994.

[6] E. Wigner, The unreasonable effectiveness of mathematics in the natural sciences, Comm. Pure Appl. Math. 13 (1960), 1-14.

[7] Mathematics matters, http://www.ima.org.uk/i_love_maths/ mathematics_matters.cfm (retrieved 2/12/2013).

[8] ThatsMaths, http://thatsmaths.com/ (retrieved 2/12/2013).

Department of Mathematics and Statistics, National University of Ireland Maynooth, Maynooth, Co. Kildare, Ireland.

E-mail address: stephen.buckley@maths.nuim.ie 